Pacific Journal of Mathematics

CONTINUOUS CANONICAL FORMS FOR MATRICES UNDER 


\title{
CONTINUOUS CANONICAL FORMS FOR MATRICES UNDER UNITARY EQUIVALENCE
}

\author{
Vern PAULSEN
}

\begin{abstract}
A continuous canonical form for the unitary equivalence of $2 \times 2$ complex matrices is constracted and it is proved that for $n \geqq 3$ there does not exist a continuous canonical form for the unitary equivalence of $n \times n$ complex matrices. Both results have applications to the study of singly generated $C^{*}$-algebras of type $I_{n}$.
\end{abstract}

1. Introduction. Let $M_{n}$ be the ring of $n \times n$ complex matrices with its usual topology. We shall call a function $J: M_{n} \rightarrow M_{n}$ a canonical form for the relation of unitary equivalence on $M_{n}$ if $J$ satisfies:

(i ) $J(A)$ is unitarily equivalent to $A$,

(ii) if $B$ is unitarily equivalent to $A$, then $J(A)=J(B)$.

In other words, a canonical form for the relation of unitary equivalence is a rule for selecting a unique matrix from each unitary equivalence class.

If the equivalence relation of similarity is considered instead of the relation of unitary equivalence, then the map which sends each matrix to its Jordan form is a well known and reasonably computable example of a canonical form for the relation of similarity. The problem of constructing a canonical form for the relation of unitary equivalence does not have as satisfactory a solution as the Jordan form yields for similarity. There are several reasons for this. First, the problem of finding suitable invariants that determine when two $n \times n$ matrices are unitarily equivalent was solved more recently than the corresponding problem for similarity. A complete set of unitary invariants for $n \times n$ complex matrices was first found by Specht [30], but the number of invariants given was infinite. Specht proved that if $W$ denotes the free multiplicative semi-group generated by the symbols $x$ and $y$, then two $n \times n$ matrices $A$ and $B$ are unitarily equivalent if and only if $\operatorname{Tr}\left(w\left(A, A^{*}\right)\right)=\operatorname{Tr}\left(w\left(B, B^{*}\right)\right)$ for all $w(x, y) \in W$, where $\operatorname{Tr}(\cdot)$ is the trace function. A finite set of invariants was given in [24, Thm. 2] where for $n$ fixed but arbitrary, a subset of $W$ containing $4^{n^{2}}$ elements which forms a complete set of unitary invariants is explicitly constructed. The sharpness of the above bound is not known, but a detailed analysis of the cases $n=2$ and $n=3$ in [21] and [23], respectively, shows that considerably fewer invariants are sufficient. The second difficulty encountered in constructing a canonical form for the relation 
of unitary equivalence is that, in general, a given matrix is unitarily equivalent to "fewer" matrices than it is similar to and hence a much "larger" set of canonical forms is needed. This is amply illustrated in [28]. Various authors have given inductive definitions of canonical forms for the relation of unitary equivalence [5], [20], and [28], but it is hard to visualize the final canonical form. Ex. plicit canonical forms are constructed in [6] and [23] for the cases $n=2$ and $n=3$, respectively.

More recent work was concerned with determining what additional properties a canonical form might satisfy. In [4, Cor. 4] it is proved that the map which associates to each matrix its Jordan form is a Borel measurable function from $M_{n}$ to $M_{n}$, and thus is a Borel measurable canonical forms for similarity. It is implicit in [2, Cor. 3.4.1] and [4] that canonical forms exist for the relation of unitary equivalence on $M_{n}$ which are Borel measurable functions. Further results concerned with performing similarity and unitary transforms in a Borel measurable or continuous fashion are applied to the study of von Neumann algebras of type $I$ in [6], [10], [11], [13], [14], [15], [17], [25], and [26].

The problem we wish to consider is whether or not canonical forms for the relation of unitary equivalence exist which are continuous. Again, when similarity is considered instead of unitary equivalence, the problem is easier and the answer is that there can be no canonical form for similarity which is continuous (cf. Remark 1). Quite surprisingly, for unitary equivalence the answer depends on $n$. We shall show that no continuous canonical form exists for $n \geqq 3$, but for $n=2$ we shall construct a continuous canonical form.

In the same way that the measurability of certain similarity and unitary transforms is useful in the study of von Neumann algebras, the continuity of unitary transforms and canonical forms for unitary equivalence has applications to the study of $C^{*}$-algebras. In fact, the original motivation for studying such continuity problems arose from questions concerning the structure of the algebras $C_{x}^{*}(Y)$ which were introduced in [29]. These algebras are pertinent to attempts to generalize the Brown-Douglas-Fillmore theory ([8] and [9]) to the class of essentially $n$-normal operators (cf. for example, [27]). The applications of the results of this paper to the algebras $C_{\chi}^{*}(Y)$ will be discussed in [22].

The main results of this paper appear in $\S 3$. To prove them we shall need some preliminaries which will allow us to deduce the existence of a continuous unitary transform from the existence of a "pointwise" unitary transform. This is the content of $\S 2$. 
2. Selection theorems, Let $X$ be a compact Hausdorff space, let $C(X)$ be the $C^{*}$-algebra of continuous complex-valued functions on $X$, and let $M_{n}(X)$ be the *-algebra of continuous functions from $X$ to $M_{n}$, where the algebraic operations are defined pointwise. If we set

$$
\|A\|=\sup _{x \in X}\|A(x)\|
$$

for $A \in M_{n}(X)$, then $M_{n}(X)$ becomes a $C^{*}$-algebra (identifiable with the $C^{*}$-algebra of all $n \times n$ matrices with entries from $C(X)$ ). When $X$ is a Stonian space (i.e., when the closure of every open set in $X$ is open), $M_{n}(X)$ is an $n$-homogeneous $A W^{*}$-algebra [18].

If $X$ is Stonian and if $A$ and $B$ are elements of $M_{n}(X)$ with $A(x)$ unitarily equivalent to $B(x)$ for each $x$ in $X$, then one knows from [25, Thm. 3] that $A$ and $B$ are unitarily equivalent as elements of $M_{n}(X)$, i.e., that there is a unitary $U$ in $M_{n}(X)$ with $U^{*} A U=B$. Various examples in [11] show that if one drops the hypothesis that $X$ be Stonian, then this result is not generally true. To establish the assertions of $\S 3$ we will need a variation of this theorem which is valid for spaces which are not Stonian. To prove such a theorem we will make some additional hypotheses and establish a theorem concerning the structure of certain *-homomorphisms of $M_{n}(X)$. In [7], [9], and [31] different versions of this structure theorem are referred to, but the version we shall present here is quite accessible and so we include its proof. We begin with some relevant definitions.

An element $A$ of $M_{n}$ is called irreducible if the only (Hermitian) projections in $M_{n}$ that commute with $A$ are the trivial ones. Equivalently, a matrix $A$ in $M_{n}$ is irreducible if and only if the algebra of all polynomials $p\left(A, A^{*}\right)$ in $A$ and $A^{*}$ is equal to $M_{n}$.

If $E$ is a nontrivial complex line bundle over a compact Hausdorff space $X$ (cf. for example, [3]), but the direct sum of $n$ copies of $E, n E$, is trivial, then we will call $E$ an $n$-torsion line bundle. We shall call $X$ n-torsion free if there does not exist an $n$-torsion line bundle over $X$.

We are now in a position to state our theorems.

THEOREM 2.1. Let $X$ be a compact, Hausdorff space, and let $\phi$ be $a^{*}$-algebra homomorphism from $M_{n}(X)$ into $M_{n}(X)$ which is elementwise center-preserving. If $X$ is n-torsion free, then there is a unitary $U$ in $M_{n}(X)$ with $\phi(A)=U^{*} A U$.

TheOREm 2.2. If $X$ is n-torsion free and if $A$ and $B$ belong to $M_{n}(X)$ with $A(x)$ and $B(x)$ unitarily equivalent, irreducible matrices for each $x$ in $X$, then $A$ and $B$ are unitarily equivalent elements of $M_{n}(X)$. 
Proof of Theorem 2.1. We shall regard $n \times n$ matrices as operators on the Hilbert space $C^{n}$ and regard elements of $M_{n}(X)$ as giving rise to an operator on $C^{n}$ for each $x$ in $X$ without further comment.

For $1 \leqq i, j \leqq n$, let $E_{i, j}$ be the element of $M_{n}(X)$ whose value at each point is the matrix which has a 1 in the $(i, j)$ th position and is 0 in all the remaining positions. If for $1 \leqq i, j \leqq n$ we set $P_{i, j}=\phi\left(E_{i, j}\right)$, then it is easy to see that for each $x$ in $X$ the set $\left\{P_{i, i}(x)\right\}_{i=1}^{n}$ is a collection of mutually orthogonal rank one projections. Furthermore, each $P_{i, j}(x)$ is a partial isometry with initial space the range of $P_{j, j}(x)$ and final space the range of $P_{i, i}(x)$. For $1 \leqq i \leqq n$, let $H_{i}$ be the subset of $X \times C^{n}$ consisting of all points $(x, v)$ with $v$ in the range of $P_{i, i}(x)$. Since each $P_{i, i}$ is continuous, this insures the local triviality of $H_{i}$ and so $H_{i}$ becomes a complex line bundle over $X$.

If $i \neq j$, then $P_{i, j}$ defines a vector bundle isomorphism from $H_{j}$ to $H_{i}$ via the mapping $(x, v) \rightarrow\left(x, P_{i, j}(x) v\right)$. Thus, $n H_{1}$ is vector bundle isomorphic to $\sum_{i=1}^{n} \oplus H_{i}$, which is in turn vector bundle isomorphic to $X \times C^{n}$. Thus, since $X$ is $n$-torsion free by hypothesis, $H_{1}$ must be a trivial bundle, and so there exists a continuous function $u_{1}$ from $X$ to $C^{n}$ such that $\left\|u_{1}(x)\right\|=1$ and such that $\left(x, u_{1}(x)\right) \in H_{1}$ for each $x$ in $X$. If for $2 \leqq i \leqq n$, we define $u_{i}$ by $u_{i}(x)=P_{i, 1}(x) u_{1}(x)$, then each $u_{i}$ is a continuous function from $X$ to $C^{n}$ with $\left\|u_{i}(x)\right\|=1$, and $\left(x, u_{i}(x)\right) \in H_{i}$ for each $x$ in $X$. If $U$ is the element of $M_{n}(X)$ whose $i$ th column is $u_{i}$, for $1 \leqq i \leqq n$, then $U$ is a unitary element of $M_{n}(X)$. We assert that $\phi(A)=U A U^{*}$ for every $A$ in $M_{n}(X)$.

To verify this assertion, notice that if $\left\{e_{k}\right\}_{k=1}^{n}$ is the standard orthonormal basis for $C^{n}$, then $U E_{i, j} e_{k}=\delta_{j, k} u_{i}$ and $P_{i, j} U e_{l}=\delta_{j, k} u_{i}$, where $\delta_{j, k}$ is the Kronecker delta. Thus, we have $U E_{i, j} U^{*}=P_{i, j}$. If $A$ is in $M_{n}(X)$, then we may write

$$
A=\sum_{i, j=1}^{n} \operatorname{Diag}\left(a_{i, j}\right) E_{i, j}
$$

where $\alpha_{i, j}$ is in $C(X)$ for $1 \leqq i, j \leqq n$ and Diag $\left(\alpha_{i, j}\right)$ is the element in the center of $M_{n}(X)$ which is the diagonal matrix having the function $\alpha_{i, j}$ for each diagonal entry. We have

$$
\begin{aligned}
\dot{\phi}(A) & =\sum_{i, j=1}^{n} \operatorname{Diag}\left(\alpha_{i, j}\right) \dot{\phi}\left(E_{i, j}\right) \\
& =\sum_{i, j=1}^{n} \operatorname{Diag}\left(\alpha_{i, j}\right) U E_{i, j} U^{*}=U A U^{*},
\end{aligned}
$$

which completes the proof of Theorem 2.1.

When $X$ is Stonian, all line bundles over $X$ are trivial (since 
we may find a finite cover of $X$ by compact, open subsets with the bundle trivial above each subset) and so Theorem 2.1 generalizes the part of [19, Thm. 3] which says that any elementwise centerpreserving *-algebra automorphism of an $n$-homogeneous $A W^{*}$-algebra is conjugation by a unitary element of that algebra.

We will now prove a lemma from which Theorem 2.2 follows.

Lemma 2.2. If $A$ and $B$ are elements of $M_{n}(X)$ such that $A(x)$ and $B(x)$ are unitarily equivalent, irreducible matrices for each $x$ in $X$, then there exists an elementwise center-preserving *algebra homomorphism of $M_{n}(X)$ into itself which maps $A$ to $B$.

Proof. For each $x$ in $X$ there exists a unitary matrix $U(x)$ with $U(x)^{*} A(x) U(x)=B(x)$. Since $A(x)$ and $B(x)$ are irreducible matrices, $U(x)$ is uniquely determined up to multiplication by a scalar unitary matrix. Let $E_{i, j}$ be defined as in the proof of Theorem 2.1 and for $1 \leqq i, j \leqq n$, define $P_{i, j}(x)=U(x)^{*} E_{i, j}(x) U(x)$. Notice that the definition of $P_{i, j}(x)$ is independent of the choice of the unitary $U(x)$ satisfying $U(x)^{*} A(x) U(x)=B(x)$.

We assert that for $1 \leqq i, j \leqq n, P_{i, j} \in M_{n}(X)$, i.e., that each $P_{i, j}$ is a continuous function of $X$. To establish this assertion, let us fix $x_{0}$ in $X$ and show that $P_{i, j}$ is continuous in a neighborhood of $x_{0}$. Since $A\left(x_{0}\right)$ is an irreducible matrix, there exist polynomials $p_{i, j}(y, z)$ in the noncommuting variables $y$ and $z, 1 \leqq i, j \leqq n$, such that $E_{i, j}\left(x_{0}\right)=p_{i, j}\left(A\left(x_{0}\right), A\left(x_{0}\right)^{*}\right)$. If we set $A_{i, j}=p_{i, j}\left(A, A^{*}\right)$ and $B_{i, j}=p_{i, j}\left(B, B^{*}\right)$ for $1 \leqq i, j \leqq n$, then $\left\{A_{i, j}\left(x_{0}\right)\right\}_{i, j=1}^{n}$ is a linearly independent set. Hence, in some neighborhood $\mathscr{V}$ of $x_{0},\left\{A_{i, j}(x)\right\}_{i, j=1}^{n}$ will be linearly independent for all $x$ in $\mathscr{Y}$. Thus we can find $d_{i, j, k, l} \in C(\mathscr{V})$ for $1 \leqq i, j, k, l \leqq n$, such that

$$
E_{i, j}(x)=\sum_{k, l=1}^{n} d_{i, j, k, l}(x) A_{k, l}(x)
$$

for all $x$ in $\mathscr{Y}$. Since $P_{i, j}(x)=U^{*}(x) E_{i, j}(x) U(x)=\sum_{k, l=1}^{n} d_{i, j, k, l}(x) B_{i, j}(x)$ for $1 \leqq i, j \leqq n$ and $x$ in $\mathscr{F}$, the functions $P_{i, j}$ are continuous at $x_{0}$.

We are now in a position to define a ${ }^{*}$-algebra homomorphism $\phi$ from $M_{n}(X)$ into itself. Recall that if $F$ is in $M_{n}(X)$, then $F=$ $\sum_{i, j=1}^{n} \operatorname{Diag}\left(f_{i, j}\right) E_{i, j}$ for certain $f_{i, j}$ in $C(X), 1 \leqq i, j \leqq n$. We define $\phi\left(F^{\prime}\right)=\sum_{i, j=1}^{n} \operatorname{Diag}\left(f_{i, j}\right) P_{i, j}$. It is easy to check that $\phi$ is a *algebra homomorphism which is elementwise center-preserving and that $\phi(A)=B$; thus the lemma is proved.

Theorem 2.2 now follows by applying Theorem 2.1 to the *-algebra homomorphism obtained in Lemma 2.2.

The hypothesis that $X$ be a topological space that is $n$-torsion 
free is not vacuous. In [1] a 2-torsion line bundle is constructed over $R P_{2}$. Furthermore, if we let $G_{n}$ be the group of *automorphisms of $M_{n}$ with the topology of pointwise convergence, then in [31] an elementwise center-preserving non-inner *-automorphism of $M_{n}\left(G_{n}\right)$ is constructed, and so $G_{n}$ has $n$-torsion. Using Theorem 2.1 it is possible to exhibit an $n$-torsion line bundle over $G_{n}$.

In order to determine when we may apply Theorem 2.1 or Theorem 2.2 we make a calculation using Chern classes which provides us with conditions a space must necessarily satisfy in order to have $n$-torsion. The author acknowledges his indebtedness to Dave Simen for introducing him to the theory of Chern classes. We refer the reader to [16, Chap. $1, \S 4]$ for results on Chern classes.

Let $X$ be locally compact, the countable union of compact subsets, and of finite topological dimension. If $E$ is a vector bundle over $X$, then the Chern class of $E, c(E)=1+\sum_{n=1}^{\infty} c_{n}(E)$, is an element of the graded ring $\sum_{n=0}^{\infty} H^{2 n}(X, Z)$, where $H^{j}(X, Z)$ is the $j$ th Čech cohomology group with integer coefficients. One knows that the value of $c(E)$ depends only on the vector bundle isomorphism class of $E$, that $c(E \oplus F)=c(E) \cdot c(F)$, and that if $E$ is a trivial bundle, then $c(E)=1$. Furthermore, if $E$ is a line bundle, then $c(E)=1+c_{1}(E)$, and $c_{1}(E)=0$ implies $E$ is a trivial line bundle. Thus, if $F$ is an $n$-torsion line bundle over $X$ we have

$$
1=c(n F)=\left(1+c_{1}(F)\right)^{n}=1+n c_{1}\left(F^{\prime}\right)+\cdots+c_{1}\left(F^{\prime}\right)^{n} .
$$

Since by the hypothesis $F$ is not trivial, $c_{1}(E) \neq 0$ and hence $c_{1}\left(F^{\prime}\right)$ must be an element of $H^{2}(X, Z)$ of order $n$. We summarize this as follows:

Proposition 2.3. Suppose that $X$ is a locally compact, $\sigma$-compact, Hausdorff space and that $X$ has finite topological dimension. If in addition $H^{2}(X, Z)$ has no elements of additive order $n$, then $X$ must be $n$-torsion free. In particular, every compact subset of $\boldsymbol{R}^{2}$ is $n$ torsion free for $n \geqq 2$.

3. Continuous canonical forms. We begin this section with our construction of a continuous canonical form for the relation of unitary equivalence on $M_{2}$. We shall use the result from [21] that two elements $A$ and $B$ of $M_{2}$ are unitarily equivalent if and only if $\operatorname{Tr}(A)=\operatorname{Tr}(B), \operatorname{Tr}\left(A^{2}\right)=\operatorname{Tr}\left(B^{2}\right)$, and $\operatorname{Tr}\left(A^{*} A\right)=\operatorname{Tr}\left(B^{*} B\right)$.

THeOREM 3.1. The function $J: M_{2} \rightarrow M_{2}$ defined by

$$
J(A)=\left(\begin{array}{ll}
\operatorname{Tr}(A) / 2 & b(A) \\
d(A) / 4 b(A) & \operatorname{Tr}(A) / 2
\end{array}\right)
$$


where

$$
\begin{aligned}
d(A)= & 2 \operatorname{Tr}\left(A^{2}\right)-\operatorname{Tr}(A)^{2} \\
b(A)= & 1 / \sqrt{2}\left[\operatorname{Tr}\left(A^{*} A\right)-|\operatorname{Tr}(A)|^{2} / 2\right. \\
& \left.+\left(\left(\operatorname{Tr}\left(A^{*} A\right)-|\operatorname{Tr}(A)|^{2} / 2\right)^{2}-|d(A)|^{2} / 4\right)^{1 / 2}\right]^{1 / 2},
\end{aligned}
$$

and $d(A) / b(A)$ is defined to be zero for all $A$ such that $b(A)=0$, is a continuous canonical form for the relation of unitary equivalence on $M_{2}$.

Proof. We begin by showing that

(1) $\operatorname{Tr}\left(A^{*} A\right)-|\operatorname{Tr}(A)|^{2} / 2 \geqq 0$, and

(2) $\quad\left(\operatorname{Tr}\left(A^{*} A\right)-|\operatorname{Tr}(A)|^{2} / 2\right)^{2}-|d(A)|^{2} / 4 \geqq 0$

for every $A$ in $M_{2}$, from which it will follow that $b(A)$ is well defined and continuous. Next, we will show that although there are matrices $A$ for which $b(A)=0$, the quotient $d / b$ can be extended continuously at such points by defining the quotient to be zero there.

If $A$ and $B$ are unitarily equivalent, then $J(A)=J(B)$ since all the terms in the definition of $J$ are preserved by unitary equivalence, which shows that $J$ has property (ii) of a canonical form. Thus to verify the inequalities (1) and (2) we may replace $A$ by any matrix unitarily equivalent to $A$. Since every matrix is unitarily equivalent to an upper triangular matrix, we may assume $A$ is upper triangular. Thus we may set

$$
A=\left(\begin{array}{cc}
z_{1} & s \\
0 & z_{2}
\end{array}\right)
$$

To simplify notation we set $\operatorname{Tr}(A)=t_{1}, \operatorname{Tr}\left(A^{2}\right)=t_{2}$, and $\operatorname{Tr}\left(A^{*} A\right)=t_{3}$. Then

$$
z_{1}+z_{2}=t_{1} \text { and } z_{1}^{2}+z_{2}^{2}=t_{2},
$$

from which it follows that

$$
\begin{aligned}
\left|z_{1}-z_{2}\right|^{2} & =\left|2 t_{2}-t_{1}^{2}\right| \text { and } \\
\operatorname{Re}\left(z_{1} \bar{z}_{2}\right) & =1 / 4\left(\left|t_{1}\right|^{2}-\left|2 t_{2}-t_{1}^{2}\right|\right) .
\end{aligned}
$$

Since

$$
t_{3}=\left|z_{1}\right|^{2}+\left|z_{2}\right|^{2}+|s|^{2},
$$

we have

$$
\begin{aligned}
t_{3}-\left|t_{1}\right|^{2} / 2= & \left|z_{1}\right|^{2}+\left|z_{2}\right|^{2}+|s|^{2} \\
& -1 / 2\left(4 \operatorname{Re}\left(z_{1} \bar{z}_{2}\right)+\left|2 t_{2}-t_{1}^{2}\right|\right) \\
= & |s|^{2}+\left|2 t_{2}-t_{1}^{2}\right| / 2 \geqq 0,
\end{aligned}
$$


and thus inequality (1) is established. To establish inequality (2), one simply squares both sides of the equation

$$
t_{3}-\left|t_{1}\right|^{2} / 2=|s|^{2}+\left|2 t_{2}-t_{1}^{2}\right| / 2
$$

and subtracts the appropriate positive terms.

Turning our attention to the quotient $d(A) / b(A)$ for $b(A) \neq 0$, we see that

$$
\begin{aligned}
|d(A)| / b(A) & \leqq \sqrt{2}\left|2 t_{2}-t_{1}^{2}\right| /\left(|s|^{2}+\left|2 t_{2}-t_{1}^{2}\right| / 2\right)^{1 / 2} \\
& \leqq 2\left|2 t_{2}-t_{1}^{2}\right|^{1 / 2}
\end{aligned}
$$

Since $b(A)=0$ implies $\left|2 t_{2}-t_{1}^{2}\right|=0$, if we define $d(A) / b(A)$ to be zero for every matrix $A$ such that $b(A)=0$, then it is clear from the above inequality that this extension of $d / b$ is continuous on all of $M_{2}$. Hence we have verified that $J$ is a continuous function from $M_{2}$ to $M_{2}$.

All that remains to complete the proof of Theorem 3.1 is to verify that $J$ satisfies property (i) of a canonical form, i.e., to show that $J(A)$ is unitarily equivalent to $A$. We accomplish this by showing that $\operatorname{Tr}(J(A))=\operatorname{Tr}(A), \operatorname{Tr}\left(J(A)^{2}\right)=\operatorname{Tr}\left(A^{2}\right)$, and $\operatorname{Tr}\left(J(A)^{*} J(A)\right)=$ $\operatorname{Tr}\left(A^{*} A\right)$. The first two calculations are straightforward and so we only do the final one, i.e., we show that $\operatorname{Tr}\left(J(A)^{*} J(A)\right)=t_{3}$. If $b(A)=0$, then

$$
\operatorname{Tr}\left(J(A)^{*} J(A)\right)=\left|t_{1}\right|^{2} / 2=t_{3} .
$$

Thus we may assume $b(A) \neq 0$, and so

$$
\begin{aligned}
\operatorname{Tr}\left(J(A)^{*} J(A)\right)=\left[2\left|t_{1}\right|^{2} b(A)^{2}+4 b(A)^{4}\right. \\
\left.+|d(A)|^{2} / 4\right] / 4 b(A)^{2} .
\end{aligned}
$$

Setting

$$
f=\left[\left(t_{3}-\left|t_{1}\right|^{2} / 2\right)^{2}-|d(A)|^{2} / 4\right]^{1 / 2},
$$

we have

$$
\begin{aligned}
\operatorname{Tr}\left(J(A)^{*} J(A)\right)= & {\left[\left|t_{1}\right|^{2}\left(t_{3}-\left|t_{1}\right|^{2} / 2+f\right)\right.} \\
& \left.+\left(t_{3}-\left|t_{1}\right|^{2} / 2+f\right)^{2}+\left(t_{3}-\left|t_{1}\right|^{2} / 2\right)^{2}-f^{2}\right] / 4 b(A)^{2} \\
= & {\left[2 t_{3}^{2}+2 d t_{3}-\left|t_{1}\right|^{2} t_{3}\right] / 4 b(A)^{2}=t_{3}, }
\end{aligned}
$$

which completes the calculation.

The definition of the function $J$ given in Theorem 3.1 is perhaps better motivated by the following observation. If $A$ is an element of $M_{2}$, then there exist at most two matrices of the form

$$
\left(\begin{array}{ll}
z & r \\
w & z
\end{array}\right)
$$


with $r$ nonnegative real, that are unitarily equivalent to $A$. It is easily checked that $J(A)$ is the unique matrix of the above form for which $r$ is a maximum.

It follows from [24, p. 1408] that the function $J$ defined in Theorem 3.1 when restricted to the $2 \times 2$ real matrices becomes a continuous canonical form for the relation of orthogonal equivalence.

We proceed now to the proof that there is no continuous canonical form for the relation of unitary equivalence on $M_{n}$ for $n \geqq 3$. We shall write $Q_{n}$ for the space of unitary equivalence classes of elements of $M_{n}$ with the induced quotient topology. We will denote the quotient map from $M_{n}$ to $Q_{n}$ by $p_{n}$. It is obvious that for a fixed $n$ the problem of finding a continuous canonical form for the relation of unitary equivalence on $M_{n}$ is equivalent to the problem of finding a continuous section of $p_{n}$, i.e., a continuous function $s_{n}$ from $Q_{n}$ to $M_{n}$ such that $s_{n} \circ p_{n}$ is the identity on $Q_{n}$. Indeed, given such a section $s_{n}$, we can define a continuous canonical form on $M_{n}$ by setting $J(A)=s_{n} \circ p_{n}(A)$ for $A$ in $M_{n}$. Similarly given a continuous canonical form $J$ we obtain a continuous section [12, VI. 1, 4.2] by defining $s_{n}=J \circ p_{n}^{-1}$.

THEOREM 3.2. If $n \geqq 3$, then there is no continuous section of the $\operatorname{map} p_{n}: M_{n} \rightarrow Q_{n}$ and thus there is no continuous canonical form for the relation of unitary equivalence on $M_{n}$.

Proof. Let $n \geqq 3$ be a fixed positive integer. In order to prove that there is no continuous section of the map $p_{n}$, it is sufficient to exhibit a subset of $Q_{n}$ such that no section of $p_{n}$ can be continuous when restricted to that subset.

With this in mind, for the closed unit disc $D$ we define $A$ and $B$ to be the elements of $M_{n}(D)$ given by:

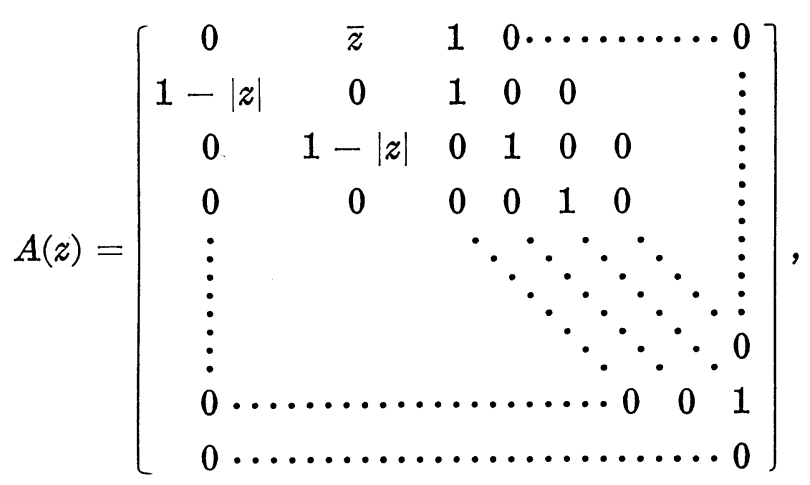




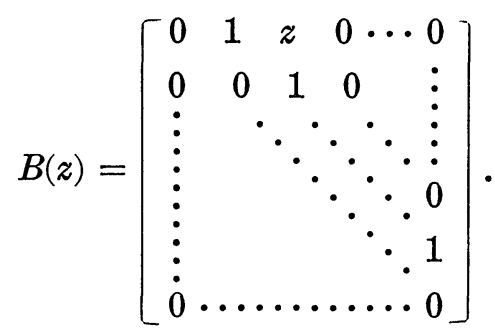

We shall show that if we define $h_{1}(z)=p_{n}(A(z))$ and $h_{2}(z)=p_{n}(B(z))$, then $h_{1}$ and $h_{2}$ are homeomorphisms of $D$ into $Q_{n}$, and furthermore $h_{1}(z)=h_{2}\left(z^{\prime}\right)$ if and only if $z=z^{\prime}$ and $|z|=1$. The union $S$ of the ranges of $h_{1}$ and $h_{2}$ is the subset on which we shall show that there can be no continuous section of $p_{n}$ defined.

Since $h_{1}$ and $h_{2}$ are continuous functions on the closed disc, to prove that they are homeomorphisms it is sufficient to prove that they are one-to-one. To this end, recall that if two matrices $P$ and $R$ are unitarily equivalent, then for any polynomial $q(x, y)$ in the noncommuting variables $x$ and $y, \operatorname{Tr}\left(q\left(P, P^{*}\right)\right)=\operatorname{Tr}\left(q\left(R, R^{*}\right)\right) . \quad \mathrm{A}$ calculation shows that $\operatorname{Tr}\left(B(z)^{*} B(z)^{2}\right)=\bar{z}$ and hence $h_{2}$ is one-to-one. Further calculations show that $\operatorname{Tr}\left(A(z)^{2}\right)=(1-|z|)(2+2 \bar{z}), \operatorname{Tr}\left(A(z)^{3}\right)=$ $3(1-|z|)^{2}$, and $\operatorname{Tr}\left(A(z)^{*} A(z)^{2}\right)=z(1-|z|)+\bar{z}$. If $h_{1}(z)=h_{1}\left(z^{\prime}\right)$, then $\operatorname{Tr}\left(A(z)^{3}\right)=\operatorname{Tr}\left(A\left(z^{\prime}\right)^{3}\right)$, and so $|z|=\left|z^{\prime}\right|$. If $|z| \neq 1$, then consideration of the equation $\operatorname{Tr}\left(A(z)^{2}\right)=\operatorname{Tr}\left(A\left(z^{\prime}\right)^{2}\right)$ shows that $z=z^{\prime}$. Similarly, when $|z|=1$, consideration of the equation $\operatorname{Tr}\left(A(z)^{*} A(z)^{2}\right)=\operatorname{Tr}\left(A\left(z^{\prime}\right)^{*} A\left(z^{\prime}\right)^{2}\right)$ shows that $z=z^{\prime}$.

If for $z$ in $D$ and $|z|=1$ we define the unitary matrix $U(z)$ by

$$
U(z)=\left[\begin{array}{cccc}
z & 0 & \cdots & 0 \\
0 & 1 & \ddots & \vdots \\
\vdots & & \ddots & 0 \\
0 & \cdots & 0 & 1
\end{array}\right]
$$

then $U(z) A(z) U(z)^{*}=B(z)$ for each $z$ in $D$ with $|z|=1$. Thus, we have $h_{1}(z)=h_{2}(z)$ when $|z|=1$. If $h_{1}(z)=h_{2}\left(z^{\prime}\right)$, then since $\operatorname{Tr}\left(B(z)^{2}\right)=0$ we must have $\operatorname{Tr}\left(A\left(z^{\prime}\right)^{2}\right)=0$, and so $\left|z^{\prime}\right|=1$. Since we must also have $\operatorname{Tr}\left(B(z)^{*} B(z)^{2}\right)=\operatorname{Tr}\left(A\left(z^{\prime}\right)^{*} A(z)^{2}\right)$, it must be the case that $z=z^{\prime}$. Thus, $h_{1}(z)=h_{2}\left(z^{\prime}\right)$ if and only if $z=z^{\prime}$ and $|z|=1$.

Let us suppose now that we have a continuous map $s$ from $S$ into $M_{n}$ such that $p_{n} \circ s$ is the identity on $S$. If we set $A_{1}(z)=s\left(h_{1}(z)\right)$ and $B_{1}(z)=s\left(h_{2}(z)\right)$, then $A_{1}$ and $B_{1}$ are elements of $M_{n}(D)$. Furthermore, for each $z$ in $D, A_{1}(z)$ and $B_{1}(z)$ are unitarily equivalent to $A(z)$ and $B(z)$, respectively. As we shall soon show, $A(z)$ and $B(z)$ (and hence $A_{1}(z)$ and $B_{1}(z)$ ) are irreducible matrices for each $z$ in $D$. Since, as is well known, $H^{2}(D, Z)=0$, by Proposition 2.3 the hypo- 
theses of Theorem 2.2 are satisfied, and thus there exist unitary elements $V$ and $W$ of $M_{n}(D)$ with $V^{*} A V=A_{1}$ and $W^{*} B W=B_{1}$. Since for $|z|=1$ we have $A_{1}(z)=B_{1}(z)$, this implies

$$
W(z) V(z)^{*} A(z) V(z) W(z)^{*}=B(z)
$$

for $|z|=1$. We define continuous functions $f$ and $g$ from the unit circle to itself by $f(z)=\operatorname{det}\left(W(z) V(z)^{*}\right)$ and $g(z)=\operatorname{det}(U(z))=z$. The Brouwer degree of $g$ is 1 , and the Brouwer degree of $f$ is zero since $f$ is homotopic to a constant function. To define the homotopy, if for $0 \leqq t \leqq 1$ and $|z|=1$ we let $F(z, t)=\operatorname{det}\left(W(t z) V(t z)^{*}\right)$, then $F(z, 1)=f(z)$ and $F(z, 0)=\operatorname{det}\left(W(0) V(0)^{*}\right)$. We also have that $U(z)^{*} W(z) V(z)^{*} A(z) V(z) W(z)^{*} U(z)=A(z)$ for $|z|=1$, and since $A(z)$ is an irreducible matrix, it must be that $U(z)^{*} W(z) V(z)^{*}$, is a scalar unitary matrix for each $z$ with $|z|=1$. But this implies that the function $\operatorname{det}\left(U(z)^{*} W(z) V(z)^{*}\right)$ from the unit circle to the unit circle has Brouwer degree a multiple of $n$. On the other hand,

$$
\operatorname{det}\left(U(z)^{*} W(z) V(z)^{*}\right)=\overline{g(z)} f(z)
$$

which has Brouwer degree -1 . This contradiction completes the proof of Theorem 3.2 except for the verification of the claim that $A(z)$ and $B(z)$ are irreducible matrices for all $z$ in $D$.

If $B(z)$ was not an irreducible matrix for some $z$ in $D$, then the Jordan form of $B(z)$ would have more than one block. If we define an element of $M_{n}(D)$ by

$$
S(z)=\left[\begin{array}{ccccc}
1 & z & 0 & \cdots & 0 \\
0 & 1 & 0 & \cdots & 0 \\
\vdots & \ddots & \ddots & \ddots & \vdots \\
\vdots & \ddots & \ddots & \ddots & 0 \\
0 & \cdots & \cdots & 0 & 1
\end{array}\right],
$$

then for all $z$ in $D, S^{-1}(z) B(z) S(z)$ is the $n \times n$ elementary Jordan block with eigenvalue zero. Hence, by the uniqueness of the Jordan form, $B(z)$ must be irreducible for all $z$ in $D$.

We shall prove that $A(z)$ is irreducible for all $z$ in $D$ by induction. To this end, for each $k, 3 \leqq k \leqq n$, let $A_{k}$ be the element of $M_{k}(D)$ obtained from $A$ by deleting the last $n-k$ rows and columns, so that

$$
A_{k+1}(z)=\left[\begin{array}{c:c} 
& 0 \\
A_{k}(z) & \vdots \\
& 1 \\
\hdashline 0 \cdots & 0
\end{array}\right] .
$$


It is possible to show by a direct calculation that the only selfadjoint matrix which commutes with $A_{3}(z)$ for any $z$ in $D$ is a scalar matrix. So by induction we may assume that for any $z$ in $D$ and $3 \leqq j \leqq k, A_{j}(z)$ is an irreducible matrix. Now let $z$ be an arbitrary but fixed point in $D$ and assume $P$ is a $(k+1) \times(k+1)$ self-adjoint matrix which commutes with $A_{k+1}(z)$. We may write

$$
P=\left[\begin{array}{c:c}
P_{k} & P_{1} \\
\hdashline P_{1}^{*} & P_{2}
\end{array}\right]
$$

with $P_{k}$ a $k \times k$ self-adjoint matrix. Since $P$ commutes with $A_{k+1}(z)$, performing the block matrix multiplication yields

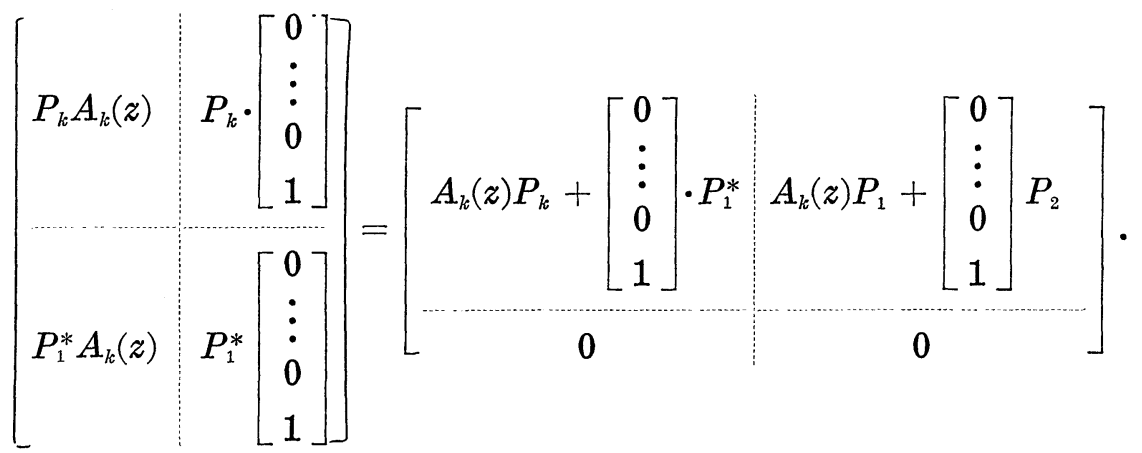

Comparing the lower right entries, we see that the last entry in $P_{1}$ must be zero. From the equation $P_{1}^{*} A_{k}(z)=0$, it now follows that $P_{1}=0$. Thus we have $P_{k} A_{k}(z)=A_{k}(z) P_{k}$ and it follows from the inductive hypotheses that $P_{k}$ must be a scalar matrix. Finally, comparing the upper right entries in the block matrices, we see that $P$ itself must be a scalar matrix. This completes the proof of the last claim and of Theorem 3.2.

\section{Remarks.}

(1) To prove that there is no continuous canonical form for the similarity of $n \times n$ matrices for $n \geqq 2$, one observes that it is possible to construct a convergent sequence of matrices $\left\{A_{k}\right\}_{k=1}^{\infty}$ with limit $A$ such that any two matrices belonging to the sequence are similar, but no matrix belonging to the sequence is similar to $A$.

(2) If we let $\mathcal{O}_{n}$ denote the set of irreducible matrices in $M_{n}$ and let $\mathscr{B}_{n}$ denote the corresponding subset of $Q_{n}$, then $\mathscr{O}_{n}$ is actually a fiber bundle over $\mathscr{B}_{n}$ with fiber equal to $G_{n}$. In this context Theorem 3.1 shows that $\mathscr{O}_{2}$ is a trivial fiber bundle, while the proof of Theorem 3.2 shows that $\mathscr{O}_{n}$ is not a trivial fiber bundle over $\mathscr{B}_{n}$ for any $n \geqq 3$. 


\section{REFERENCES}

1. J. F. Adams, Vector fields on spheres, Ann. Math., 75 (1962), 603-632.

2. W. Arveson, An Invitation to $C^{*}$-algebras, Springer-Verlag, New York, 1976.

3. M. F. Antiyah, K-theory, W. A. Benjamin, New York, 1967.

4. E. A. Azoff, Borel measurability in linear algebra, Proc. Amer. Math. Soc., 42 (1974), 346-350.

5. J. Brenner, The problem of unitary equivalence, Acta Math., 86 (1951), 297-308.

6. A. Brown, Unitary Equivalence of binormal operators, Amer. J. Math., 76 (1954), 414 434 .

7. L. Brown, Extensions and the structure of $C^{*}$-algebras, preprint.

8. L. Brown, R. Douglas, and P. Fillmore, Unitary equivalence modulo the compact operators and extensions of $C^{*}$-algebras, Lecture Notes in Math., Vol. 345, SpringerVerlag, New York, 1973, 55-128.

9. - Extensions of $C^{*}$-algebras and K-homology, preprint.

10. D. Deckard and C. Pearcy, On matrices over the ring of continuous complex-valued functions on a Stonian space, Proc. Amer. Math. Soc., 14 (1963), 322-328.

11. - On continuous matrix-valued functions on a Stonian space, Pacific J. Math., 14 (1963), 857-869.

12. J. Dugundji, Topology, Allyn and Bacon, Boston, 1966.

13. S. R. Foguel, Normal operators of finite multiplicity, Comm. Pure Appl. Math., 11 (1958), 297-313.

14. H. Gonshor, Spectral theory for a class of nonnormal operators I, Canad. J. Math., 8 (1956), 449-461.

15. H. Gonshor, Spectral theory for a class of nonnormal operators II, Canad. J. Math., 10 (1958), 97-102.

16. F. Hirzebruch, Topological methods in algebraic geometry, 3rd ed. Die Grundlehren der Mathematischen Wissenschaften, Vol. 131, Springer-Verlag, New York, 1966.

17. T. B. Hoover, Hyperinvariant subspaces for n-normal operators, Acta. Sci. Math. (Szeged), 32 (1971), 109-119.

18. I. Kaplansky, Projections in Banach algebras, Ann. of Math., 53 (1951), 235-249.

19. - Algebras of type I, Ann. of Math., 56 (1952), 460-472.

20. D. E. Littlewood, On unitary equivalence, J. London Math. Soc., 28 (1953), 314-322.

21. F. D. Murnaghan, On the unitary invariants of a square matrix, An. Acad. Brasil. Ci., 26 (1954), 1-7.

22. V. Paulsen, Weak compalence invariants for essentially n-normal operators, in preparation.

23. C. Pearcy, A complete set of unitary invariants for $3 \times 3$ complex matrices, Trans. Amer. Math. Soc., 104 (1962), 425-429.

24. - A complete set of unitary invariants for operators generating finite $W^{*}$ algebras of type I, Pacific J. Math., 12 (1962), 1405-1416.

25. - On unitary equivalence of matrices over the ring of continuous complexvalued functions on a Stonian space, Canad. J. Math., 15 (1963), 323-331.

26. C. Pearcy and J. Ringrose, Trace preserving isomorphisms in finite operator algebras, Amer. J. Math., 90 (1968), 444-455.

27. C. Pearcy and N. Salinas, Extensions of $C^{*}$-algebras and the reducing essential matricial spectra of an operator, K-theory and operator algebras, Springer-Verlag Lecture Notes in Mathematics, Vol. 575, 96-112.

28. H. Radjavi, On unitary equivalence of arbitrary matrices, Trans. Amer. Math. Soc., 104 (1962), 363-373.

29. N. Salinas, Extensions of $C^{*}$-algebras and essentially n-normal operators, Bull. Amer. Math. Soc., 82 (1976), 143-149.

30. W. Specht, Zur Theories der Matrizen. II, Jber. Deutsch. Math. Verein., 50 (1940), 19-23. 
31. J. Tomiyama and M. Takesaki, Applications of fibre bundles to the certain class of $C^{*}$-algebras, Tôhoku Math. J., (2), 13 (1961), 498-522.

Received May 9, 1977 and in revised form September 23, 1977. This is part of the author's $\mathrm{Ph}$. D. thesis written at the University of Michigan under the direction of Professor C. Pearcy.

UNIVERSITY OF KANSAS

LAWRENCE, KS 66045 


\section{PACIFIC JOURNAL OF MATHEMATICS}

\section{EDITORS}

RICHARD ARENS (Managing Editor)

University of California

Los Angeles, California 90024

C. W. CURTIS

University of Oregon

Eugene, OR 97403

C. C. MOORE

University of California

Berkeley, CA 94720

\section{J. DUGUNDJI}

Department of Mathematics University of Southern California Los Angeles, California 90007

R. Finn aNd J. Milgram Stanford University Stanford, California 94305

\section{ASSOCIATE EDITORS}

E. F. BeCKenbaCH

B. H. NeumanN

F. WOLF

K. YosHIDA

\section{SUPPORTING INSTITUTIONS}

UNIVERSITY OF BRITISH COLUMBIA CALIFORNIA INSTITUTE OF TECHNOLOGY UNIVERSITY OF CALIFORNIA MONTANA STATE UNIVERSITY UNIVERSITY OF NEVADA, RENO NEW MEXICO STATE UNIVERSITY OREGON STATE UNIVERSITY UNIVERSITY OF OREGON
UNIVERSITY OF SOUTHERN CALIFORNIA STANFORD UNIVERSITY UNIVERSITY OF HAWAII UNIVERSITY OF TOKYO UNIVERSITY OF UTAH WASHINGTON STATE UNIVERSITY UNIVERSITY OF WASHINGTON 


\section{Pacific Journal of Mathematics}

\section{Vol. 76, No. $1 \quad$ November, 1978}

Ata Nuri Al-Hussaini, Potential operators and equimeasurability ......... 1

Tim Anderson and Erwin Kleinfeld, Semisimple nil algebras of type $\delta . \ldots .99$

Stephen LaVern Campbell, Linear operators for which $T^{*} T$ and $T+T^{*}$

commute. III ......................................

Robert Jay Daverman, Special approximations to embeddings of codimension one spheres...............................

Donald M. Davis, Connective coverings of $\mathrm{BO}$ and immersions of projective

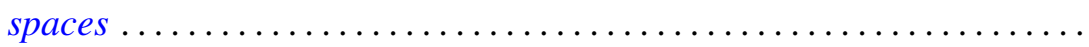

V. L. (Vagn Lundsgaard) Hansen, The homotopy type of the space of maps of

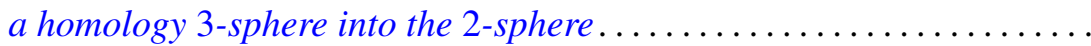

James Victor Herod, A product integral representation for the generalized

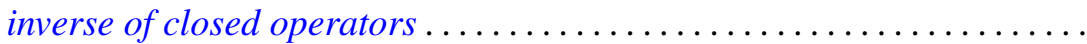

A. A. Iskander, Definability in the lattice of ring varieties ..............

Russell Allan Johnson, Existence of a strong lifting commuting with a

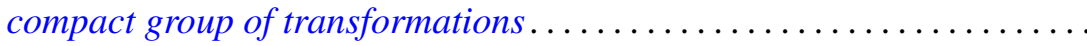

Heikki J. K. Junnila, Neighbornets...................... 83

Klaus Kalb, On the expansion in joint generalized eigenvectors . ......... 109

F. J. Martinelli, Construction of generalized normal numbers . . . . . . . . . 117

Edward O'Neill, On Massey products ....................... 123

Vern Ival Paulsen, Continuous canonical forms for matrices under unitary

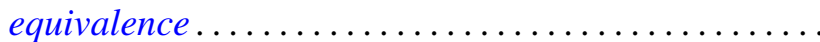

Justin Peters and Terje Sund, Automorphisms of locally compact groups . . . 143

Duane Randall, Tangent frame fields on spin manifolds . . . .

Jeffrey Brian Remmel, Realizing partial orderings by classes of co-simple sets . . . .

J. Hyam Rubinstein, One-sided Heegaard splittings of 3-manifolds ...

Donald Charles Rung, Meier type theorems for general boundary approach

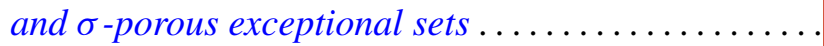

Ryōtarō Satō, Positive operators and the ergodic theorem

Ira H. Shavel, A class of algebraic surfaces of general type constructed from

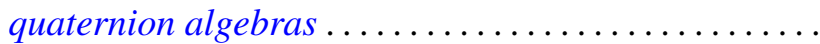

Patrick F. Smith, Decomposing modules into projectives and injectives ....

Sergio Eduardo Zarantonello, The sheaf of outer functions in the polydisc... 